\title{
Anticorps \\ monoclonaux \\ biosimilaires
}

Étude comparative

des qualités analytique et fonctionnelle

> La mise sur le marché de biosimilaires requiert une démonstration stricte de la similarité avec l'anticorps de référence, au travers d'études précliniques et cliniques. Cet article synthétise l'ensemble des analyses physicochimiques et fonctionnelles mises en œuvre in vitro, préalables à la réalisation d'études cliniques. Pour chaque caractéristique critique de l'anticorps, nous avons détaillé les techniques analytiques communément employées, leur principe de fonctionnement, ainsi que le type d'informations que ces techniques permettent d'obtenir. <

L'agence européenne des médicaments (EMA) et l'Union européenne (UE) ont joué un rôle pionnier dans la réglementation des médicaments biosimilaires en mettant en place un cadre solide pour leur approbation et en influençant leur développement à l'échelle mondiale, y compris pour les anticorps monoclonaux (AcM) et les protéines de fusion impliquant la région Fc des immunoglobulines (Ig) [1]. L'approbation par l'UE du premier biosimilaire de la somatropine (Omnitrope), en 2006, a été suivie par celles des biosimilaires de protéines et de glycoprotéines plus complexes, puis des premiers biosimilaires d'AcM dès 2013 [Remsima ${ }^{\circledR}$ et Inflectra ${ }^{\circledR}$, biosimilaires de l'infliximab (Remicade ${ }^{\circledR}$ )] [2]. Parallèlement, les professionnels de santé ont pu approfondir leur expérience concernant leur utilisation. Aujourd'hui, ces médicaments font partie intégrante de l'offre de traitements biologiques efficaces dans l'UE tout en présentant des garanties adéquates concernant la sécurité des patients. Actuellement, près de 30 biosimilaires d'AcM et de protéines de fusion ont été approuvés en Europe [adalimumab (8), bévacizumab (2), étanercept (2), infliximab (4), rituximab (6) et trastuzumab (5), voir Tableau /]. II s'agit de copies des premières générations d'AcM approuvés entre 1997 et 2005 , dont les brevets princeps ont expiré et dont les chiffres d'affaires annuels figurent dans le top 15 des médicaments (de 7 à 19 milliards de dollars). Seul le cétuximab, qui cible le récepteur de l'EGF (epidermal growth factor) manque pour l'instant à cette liste, pro-

\author{
Alain Beck ${ }^{1}$, Davy Guillarme ${ }^{2}$, \\ Sandrine Fleury-Souverain ${ }^{2}$, \\ Elsa Bodier-Montagutelli ${ }^{3,4}$, Renaud Respaud ${ }^{3,4}$
}

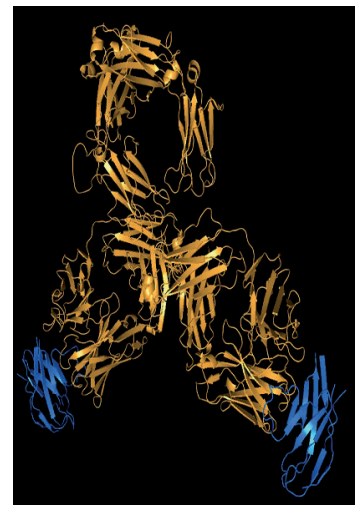

${ }^{1}$ IRPF, Centre d'Immunologie Pierre Fabre (CIPF), 5 avenue Napoléon III, 74160 Saint-Julien en Genevois, France.

${ }^{2}$ École des Sciences Pharmaceutiques,

Université of Genève, Université de Lausanne, CMU - Rue MichelServet, 1, 1206 Genève, Suisse. ${ }^{3}$ Université FrançoisRabelais de Tours, UMR 1100, CHRU de Tours, Service de pharmacie,

bablement en raison de la présence F-37032 Tours, France.

${ }^{4}$ Inserm, Centre d'étude des pathologies respiratoires, UMR 1100, F-37032 Tours, France. renaud.respaud@univ-tours.fr dans la partie Fab de l'immunoglobuline qui rend la démonstration de biosimilarité plus difficile $[3,4]$.

Par rapport aux molécules chimiques de petite taille (entre 150 et $500 \mathrm{Da}$ ), les médicaments biologiques de type AcM sont des macromolécules de structures très complexes (150000 Da) [5] $(\rightarrow)$.

$(\rightarrow)$ Voir la Synthèse de A. Beck et al., $\mathrm{m} / \mathrm{s}$ $n^{\circ} 12$, décembre 2009, page 1024

Par définition, les AcM biosimilaires sont dotés de propriétés physiques, chimiques et biologiques très semblables à celles des anticorps de références approuvés dans l'UE [6]. Tout comme pour les AcM originaux, de légères différences (micro-hétérogénéités ou protéoformes) sont tolérées, à condition qu'elles ne soient pas cliniquement significatives en termes de sécurité ou d'efficacité [7]. La marge de variabilité autorisée pour un AcM biosimilaire est la même que celle autorisée entre les lots de l'AcM de référence [8]. Le respect de cette marge est assuré par un procédé de fabrication robuste et validé, permettant de garantir que tous les lots du médicament présentent les spécifications requises, ainsi que par un ensemble de plus de 50 méthodes de contrôles de qualité (Tableau II) que nous discuterons.

Des études de comparabilité de la qualité analytique et fonctionnelle [9], non cliniques (pharmacodynamique et toxicologique) [10] et cliniques sont la pierre angulaire du développement d'anticorps biosimilaires [11]. 


\begin{tabular}{|c|c|c|c|c|c|c|}
\hline \multirow{2}{*}{$\begin{array}{l}\text { Dénomi- } \\
\text { nation } \\
\text { commune } \\
\text { interna- } \\
\text { tionale } \\
\text { (cible } \\
\text { antigé- } \\
\text { nique) }\end{array}$} & \multicolumn{4}{|c|}{ Médicament de référence } & \multicolumn{2}{|c|}{ Médicaments biosimilaires } \\
\hline & Nom & $\begin{array}{l}\text { AMM } \\
\text { États- } \\
\text { Unis / } \\
\text { Euro- } \\
\text { péenne }\end{array}$ & Indications thérapeutiques & $\begin{array}{l}\text { Chiffres } \\
\text { d'af- } \\
\text { faire } \\
2017 *\end{array}$ & Noms & $\begin{array}{c}\text { Dates } \\
\text { AMM } \\
\text { Euro- } \\
\text { péenne** }\end{array}$ \\
\hline $\begin{array}{l}\text { Adalimumab } \\
(\mathrm{TNF}-\alpha)\end{array}$ & Humira & $2003 / 2002$ & $\begin{array}{l}\text { Spondylarthrite ankylosante } \\
\text { Maladie de Crohn } \\
\text { Arthrite chronique juvénile } \\
\text { Psoriasis } \\
\text { Rhumatisme psoriasique } \\
\text { Polyarthrite rhumatoïde } \\
\text { Rectocolite hémorragique }\end{array}$ & $\begin{array}{l}18937 \text { mil- } \\
\text { liards US\$ } \\
\left(n^{\circ} 1\right)\end{array}$ & $\begin{array}{l}8 \text { biosimilaires: } \\
\text { Amgevita } \\
\text { Cyltezo } \\
\text { Halimatoz } \\
\text { Hefiya } \\
\text { Hulio } \\
\text { Hyrimoz } \\
\text { Imraldi } \\
\text { Solymbic }\end{array}$ & $\begin{array}{l}2017 \\
2017 \\
2018 \\
2018 \\
2018 \\
2017 \\
2017 \\
2017\end{array}$ \\
\hline $\begin{array}{l}\text { Bévacizumab } \\
\text { (VEGF-A) }\end{array}$ & Avastin & $2005 / 2004$ & $\begin{array}{l}\text { Cancer du sein } \\
\text { Cancer des trompes de Fallope } \\
\text { Cancer bronchique non à petites cellules } \\
\text { Cancer de l'ovaire } \\
\text { Cancer péritonéal } \\
\text { Cancer du rein }\end{array}$ & $\begin{array}{l}7042 \text { mil- } \\
\text { liards de } \\
\text { dollars } \\
\left(n^{\circ} 6\right)\end{array}$ & $\begin{array}{l}2 \text { biosimilaires: } \\
\text { Mvasi } \\
\text { Zirabev }\end{array}$ & $\begin{array}{l}2018 \\
2019\end{array}$ \\
\hline $\begin{array}{l}\text { Etanercept } \\
(\mathrm{TNF}-\alpha)\end{array}$ & Enbrel & $1998 / 2000$ & $\begin{array}{l}\text { Spondyloarthrite axiale } \\
\text { Rhumatisme psoriasique } \\
\text { Psoriasis en plaques } \\
\text { Polyarthrite rhumatoïde }\end{array}$ & $\begin{array}{l}8345 \text { mil- } \\
\text { liards US\$ } \\
\left(n^{\circ} 2\right)\end{array}$ & $\begin{array}{l}2 \text { biosimilaires: } \\
\text { Benepali } \\
\text { Erelzi }\end{array}$ & $\begin{array}{l}2016 \\
2017\end{array}$ \\
\hline $\begin{array}{l}\text { Infliximab } \\
(T N F-\alpha)\end{array}$ & $\begin{array}{l}\text { Remi- } \\
\text { cade }\end{array}$ & $1999 / 1998$ & $\begin{array}{l}\text { Spondylarthrite ankylosante } \\
\text { Maladie de Crohn } \\
\text { Psoriasis } \\
\text { Rhumatisme psoriasique } \\
\text { Polyarthrite rhumatoïde } \\
\text { Rectocolite hémorragique }\end{array}$ & $\begin{array}{l}7772 \text { mil- } \\
\text { liards US\$ } \\
\left(n^{\circ} 4\right)\end{array}$ & $\begin{array}{l}4 \text { biosimilaires: } \\
\text { Flixabi } \\
\text { Inflectra } \\
\text { Remsima } \\
\text { Zessly) }\end{array}$ & $\begin{array}{l}2016 \\
2013 \\
2013 \\
2018\end{array}$ \\
\hline $\begin{array}{l}\text { Rituximab } \\
(C D 20)\end{array}$ & $\begin{array}{l}\text { Rituxan/ } \\
\text { Mabthera }\end{array}$ & $1997 / 1998$ & $\begin{array}{l}\text { Lymphome non-hodgkinien } \\
\text { Leucémie lymphoïde chronique à cellules B } \\
\text { Granulomatose avec polyangéite } \\
\text { Polyangéite microscopique } \\
\text { Polyarthrite rhumatoïde }\end{array}$ & $\begin{array}{l}7783 \text { mil- } \\
\text { liards US\$ } \\
\left(n^{\circ} 3\right)\end{array}$ & $\begin{array}{l}6 \text { biosimilaires: } \\
\text { Blitzima } \\
\text { Ritemvia } \\
\text { Rituzena, ex- } \\
\text { Tuxella } \\
\text { Rixathon } \\
\text { Riximyo } \\
\text { Truxima }\end{array}$ & $\begin{array}{l}2017 \\
2017 \\
2017 \\
2017 \\
2017 \\
2017\end{array}$ \\
\hline $\begin{array}{l}\text { Trastuzumab } \\
\text { (H\&R2/neu) }\end{array}$ & $\begin{array}{l}\text { Hercep- } \\
\text { tine/ } \\
\text { Hercep- } \\
\text { tin }\end{array}$ & $2000 / 1998$ & $\begin{array}{l}\text { Cancer du sein précoce } \\
\text { Cancer du sein métastatique } \\
\text { Cancer gastrique métastatique }\end{array}$ & $\begin{array}{l}7392 \text { mil- } \\
\text { liards US\$ } \\
\left(n^{\circ} 5\right)\end{array}$ & $\begin{array}{l}5 \text { biosimilaires : } \\
\text { Herzuma } \\
\text { Kanjinti } \\
\text { Ogivri } \\
\text { Ontruzant } \\
\text { Trazimera }\end{array}$ & $\begin{array}{l}2018 \\
2018 \\
2019 \\
2017 \\
2018\end{array}$ \\
\hline
\end{tabular}

Tableau I. Anticorps et protéine de fusion-Fc approuvés par l'EMA (références et biosimilaires, classement alphabétique). Sources : *Chiffre d'affaires 2017 d'anticorps princeps (La Merie Publishing, 9 mars 2018 ; info@lamerie.com). **Biosimilaires approuvés en Europe, www.ema.europa. eu. AMM : autorisation de mise sur le marché. 
Comme indiqué dans le Tableau //, de nombreux attributs critiques de qualité (physico-chimique et biologique) doivent être comparés avec plusieurs lots de médicaments de référence. Les propriétés physicochimiques peuvent être classées en plusieurs catégories allant de caractéristiques très simples (concentration, couleur, osmolalité, pH) que l'on retrouve également pour les petites molécules, à des éléments bien plus spécifiques des AcM, telles que leurs structures primaires, secondaires et tertiaires, la présence d'agrégats, ou les modifications post-traductionnelles. Étant donné l'extrême complexité des AcM, plusieurs méthodes analytiques orthogonales sont souvent mises en œuvre pour un seul et même attribut critique de qualité (Tableau II), afin de pouvoir tirer des conclusions solides.

\section{La structure primaire de I’AcM biosimilaire}

Lors des études de comparabilité exhaustives avec le médicament de référence, une des premières caractéristiques à prendre en compte est la structure primaire de l'AcM biosimilaire. Pour cela, un certain nombre d'informations (masse molaire de l'AcM, masse molaire des chaînes légères et lourdes, modifications post-traductionnelles) peuvent être obtenues à l'échelle de la protéine intacte en utilisant la chromatographie en phase liquide couplée à la spectrométrie de masse (LC-MS). Pour obtenir des informations sur la séquence d'acides aminés, l'appariement des ponts disulfures, ou la structure et la localisation des glycanes, il est de plus nécessaire de digérer l'AcM à l'aide d'enzymes (trypsine ou endoprotéase Lys-C, par exemple) en peptides de 0,5 à $6 \mathrm{kDa}$, qui sont ensuite analysés par LC-MS/MS. Cette approche également connue sous le nom de cartographie peptidique a aujourd'hui presque totalement remplacé les méthodes anciennes de séquençage des protéines, longues, fastidieuses et coûteuses, comme la dégradation d'Edman. Ainsi, des cartes peptidiques ont été réalisées pour deux AcM ou une protéine de fusion de référence et leurs biosimilaires (rituximab [12,13], adalimumab [14] et étanercept [15]). Dans ces études de comparabilité, la séquence d'acides aminés a été validée pour les biosimilaires du rituximab et de l'adalimumab, avec une couverture de séquence très proche ou égale à $100 \%$. Au contraire, dans le cas de l'étanercept, les cartes peptidiques ont démontré des substitutions d'acides aminés pour certaines copies du médicament de référence, qui ne peuvent donc pas être approuvées en Europe.

\section{Glycosylation de l'AcM biosimilaire}

Une autre caractéristique importante des AcM est leur glycosylation, avec en particulier l'identification et le profilage des différents types de glycanes et la détermination du site de leur $\mathrm{N}$-glycosylation. Bien que représentant seulement $2-3 \%$ de la masse totale des AcM, ces glycanes jouent un rôle capital dans l'activité biologique, la pharmacocinétique, la toxicité ou l'immunogénicité du produit. La nature et la quantité des glycanes retrouvés dans les AcM dépendent des conditions de production du produit biopharmaceutique $[16,17]$. Ces structures glucidiques peuvent être très hétérogènes, et jusqu'à une centaine de glycanes différents peuvent être retrouvés dans les AcM. D’un point de vue physico-chimique, il s'agit d'espèces très polaires, souvent électriquement neutres et dépourvues de chromophores. Afin de les rendre compatibles avec des techniques séparatives et une détection spectrophotométrique, les glycanes sont libérés par clivage enzymatique puis dérivés chimiquement (par exemple avec du 2-aminobenzamide, 2-AB), avant d'être analysés par chromatographie d'interaction hydrophile (HILIC) couplée à un détecteur de fluorescence (FD). Ce processus, relativement long mais automatisable, est aujourd'hui reconnu comme méthode de référence pour le profilage des glycanes d'un AcM.

De nombreuses autres méthodes ont également été développées pour ces molécules, dont l'électrophorèse capillaire de zone (CZE) couplée à la détection par fluorescence induite par laser (LIF) après marquage par l'aminopyrène trisulfonate (APTS). Dans la littérature traitant de la comparaison des $A c M$ de référence à leurs biosimilaires, la méthode HILIC-FD (2-AB) est très largement utilisée. Elle $a$, par exemple, permis de mettre en évidence que la nature des glycanes observés était identique entre le rituximab de référence et l'un de ses biosimilaires [12]. Des différences mineures de proportions des glycanes ont parfois été relevées pour ce rituximab biosimilaire, mais sans impact sur l'efficacité ou la toxicité du produit. Dans le cas de l'étanercept, des différences significatives ont été notées entre les profils de glycosylation de la molécule de référence et quatre de ses biosimilaires vendus sur le marché asiatique et sud-américain (nouveaux $\mathrm{N}$-glycanes, quantités différentes de glycanes sialylés [15]). Les auteurs ont logiquement conclu que ces biosimilaires d'étanercept n'étaient pas comparables à la molécule de référence.

\section{La nature et la quantité des variants}

La nature et la quantité des variants (oxydation, déamidation, isomérisation, modification sur la partie $\mathrm{N} / \mathrm{C}$-terminale, par exemple) observées dans les biosimilaires d'AcM doivent également être évaluées et comparées aux micro-variants du produit de référence en utilisant différentes méthodes analytiques orthogonales (Tableau II). La chromatographie d'échange de cations (CEX) est l'une des méthodes les plus intéressantes, car les modifications que nous avons mentionnées induisent des variations dans la charge des AcM et peuvent être directement déterminées à l'échelle de la protéine intacte. Dans la CEX, les variants acides et basiques sont élués respectivement avant et après l'isoforme principale. Certaines de ces modifications, en particulier la déamidation d'asparagines, ou l'isomérisation d'acides aspartiques, sont communes et 


\begin{tabular}{lll}
\hline $\begin{array}{l}\text { Caracté- } \\
\text { ristiques }\end{array}$ & Techniques analytiques & Paramètres analysés \\
\hline
\end{tabular}

\section{Caractérisation physico-chimique}

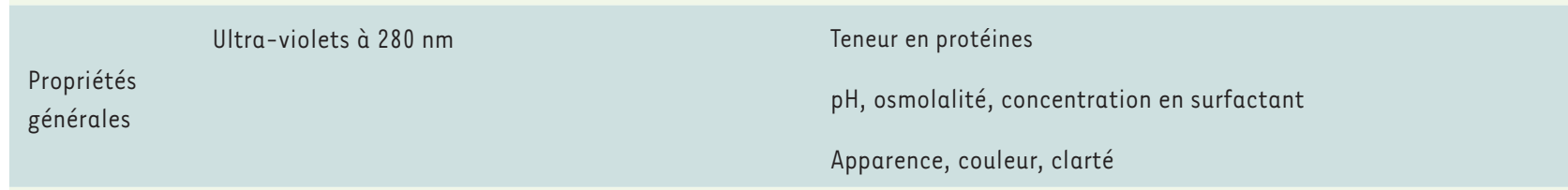

Analyse de la protéine intacte par chromatographie en phase liquide - spectrométrie de masse (LC-MS)

Analyse des sous-unités protéiques par LC-MS

Structure primaire

Modifications post-traductionnelles enzymatiques (glycosylation)

$\begin{array}{ll} & \text { Dence induite par laser (LIF) (APTS) } \\ & \text { Dichroïsme circulaire } \\ \begin{array}{l}\text { Structures } \\ \text { d'ordres } \\ \text { supérieurs }\end{array} & \text { Fluorescence (intrinsèque) } \\ & \text { Calorimétrie différentielle à balayage (DSC) } \\ & \text { Chromatographie d'exclusion stérique (SXC) } \\ & \text { Diffusion dynamique de la lumière (DLS) } \\ \text { Particules } & \text { Fractionnement par couplage flux-force asymétrique } \\ \text { et agrégats } & \text { (A4F)-LS } \\ & \text { Ultracentrifugation analytique (AUC) } \\ & \text { Obscuration de la lumière (LO) }\end{array}$

Substances apparentées et impuretés
Carte peptidique par LC-MS/MS

Focalisation isoélectrique capillaire par image (ic|EF)

Chromatographie + réaction ninhydrine

Carte peptique/glycopeptidique par LC-MS/MS

Chromatographie d'interaction hydrophile

(HILIC)-MS/MS

HILIC-FD (2-AB)

Électrophorèse capillaire de zone (CZE) - fluores-

Electrophorèse capillaire sur gel en milieu dénaturant (CGE-SDS)

Chromatographie d'échange de cations (CEX)

Focalisation isoélectrique capillaire par image (ic|દF)
Masse moléculaire de la protéine intacte

Masse moléculaire des $\mathrm{LC}$ et $\mathrm{HC}$ réduites, déglycosylées/glycosylées

Séquence protéique (carte peptidique après réduction)

Structure des ponts disulfures (carte peptidique sans réduction)

Masse moléculaire de la protéine intacte

Masse moléculaire des $\mathrm{LC}$ et $\mathrm{HC}$ réduites, déglycosylées/glycosylées

Niveau de glycation

Point isoélectrique

Composition en acides aminés

Site d'occupation des $\mathrm{N}$-glycanes

Identification des $\mathrm{N}$-glycanes

Profilage des $\mathrm{N}$-glycanes

Profilage des $\mathrm{N}$-glycanes

Structures secondaire et tertiaire

Structure secondaire

Structure tertiaire

Propriétés thermodynamiques, stabilité thermique, identification des transitions thermiques

Variants de taille de haut poids moléculaire (HMWS) et de bas poids moléculaire (LMWS)

Particules submicroniques

Particules submicroniques

Profil des agrégats

Particules sub-visibles

Variants de taille

Variants de taille

Variants de charge, distribution des isoformes (déamidation, isomérisation, parties terminales $\mathrm{N} / \mathrm{C}$ ) 


\begin{tabular}{|c|c|c|}
\hline $\begin{array}{l}\text { Substances } \\
\text { apparentées } \\
\text { et impuretés }\end{array}$ & Carte peptidique par LC-MS/MS & $\begin{array}{l}\text { Variants de charge, distribution des isoformes (déamidation, isomé- } \\
\text { risation, parties terminales } \mathrm{N} / \mathrm{C} \text { ) } \\
\text { Oxydation }\end{array}$ \\
\hline $\begin{array}{l}\text { Impuretés } \\
\text { des cellules- } \\
\text { hôtes }\end{array}$ & $\begin{array}{l}\text { ELISA } \\
\text { 2D-LC-MS (avec chromatographie d'affinité) } \\
\text { qPCR }\end{array}$ & $\begin{array}{l}\text { Protéines de cellules-hôtes } \\
\text { ADN résiduel }\end{array}$ \\
\hline \multicolumn{3}{|c|}{ Caractérisation biologique } \\
\hline $\begin{array}{l}\text { Liaison du } \\
\text { Fab et acti- } \\
\text { vité }\end{array}$ & ELISA, Résonance plasmonique de surface (SPR) & $\begin{array}{l}\text { Affinité antigène-anticorps } \\
\text { Cytotoxicité cellulaire dépendante des anticorps ( } A D C C) \text {, cytotoxicité } \\
\text { dépendante du complément ( } C D C) \text {, phagocytose dépendante des } \\
\text { anticorps }(A D C P)\end{array}$ \\
\hline $\begin{array}{l}\text { Liaison de la } \\
\text { région du Fc }\end{array}$ & $\begin{array}{l}\text { SPR } \\
\text { Tests cellulaires }\end{array}$ & 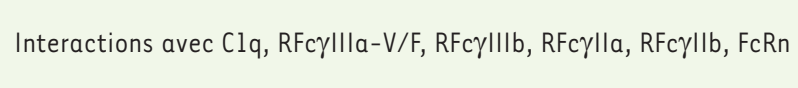 \\
\hline
\end{tabular}

Tableau II. Études comparatives de la qualité analytique (propriétés physico-chimiques) et fonctionnelles (activité biologique / pharmacologique) : anticorps de références vs biosimilaires (d'après [21-23]).

peuvent être particulièrement critiques en termes d'efficacité ou de toxicité. À titre d'exemple, dans le cas d'un biosimilaire de l'adalimumab, il a été démontré que les variants acides et basiques, bien que de même nature, étaient présents en proportions différentes entre le produit de référence et son biosimilaire (lysines C-terminales et glycanes sialylés respectivement pour les variants basiques et acides) [14]; ces différences étaient toutefois dépourvues d'impact sur la qualité du produit et donc non critiques.

\section{Les agrégats}

Une autre caractéristique importante des AcM est la quantité d'agrégats, qui correspond à l'association de plusieurs molécules protéiques conduisant à la formation de dimères, trimères, voire de particules de taille plus importante (subvisibles et visibles). Ces espèces sont connues pour être responsables d'une éventuelle toxicité du produit (immunogénicité en particulier) lorsqu'elles sont présentes en quantité trop importante. Différentes techniques peuvent être utilisées pour caractériser l'agrégation (Tableau II). Parmi elles, la chromatographie d'exclusion stérique ( $S E C$ ), qui permet de séparer et de quantifier des variants selon leur taille, est sans aucun doute la plus utilisée en contrôle qualité du fait de sa simplicité, sa rapidité et son coût modéré. Dans le cas du rituximab, un des biosimilaires testés dans l'étude de Nupur et al. présentait une quantité d'agrégats supérieure au produit de référence $(1,9 \%$ vs environ $0,5 \%$ ), mais toujours dans les spécifications établies (inférieure à $2 \%$ ) [13]. Le taux d'agrégats du biosimilaire de l'adalimumab testé par Lee et al. était également compris dans les limites d'acceptabilité (0,5\% maximum) [14]. Cinq des sept biosimilaires de l'étanercept évalués avaient des profils SEC non comparables avec la molécule de référence [15], suggérant un faible niveau de qualité ainsi qu'un risque potentiel pour le patient.

\section{Structures secondaires, tertiaires et quaternaires}

Il est également important de contrôler les structures secondaires, tertiaires et quaternaires des protéines (structures d'ordre supérieur), qui peuvent impacter leur activité biologique et/ou leur toxicité. Pour cette raison, ces dernières doivent être caractérisées dans le cas d'un médicament biosimilaire et comparées au médicament de référence. Différentes techniques spectroscopiques peuvent être utilisées (Tableau II). Parmi ces approches, la plus communément utilisée est le dichroïsme circulaire (CD), dont le principe de base est d'évaluer comment une molécule optiquement active (cas des protéines) absorbe la lumière selon que sa polarisation est circulaire droite ou gauche. En utilisant une détection par ultra-violets (UV) lointains (180-250 nm), la structure secondaire (pourcentage d'hélices alpha, de feuillets bêta, etc.) peut être déterminée, alors que la structure tertiaire (localisation des acides aminés aromatiques - phénylalanine, tyrosine, tryptophane - et des ponts disulfures) est accessible avec une détection par UV proche $(250-350 \mathrm{~nm})$. À titre d'exemple, cette approche a été utilisée avec succès pour démontrer l'équivalence des structures secondaires et tertiaires pour un biosimilaire de l'adalimumab [14]. Les résultats obtenus pour la structure secondaire ont permis de confirmer que les feuillets bêta dominaient dans la structure des AcM, et ont également été confirmés par spectroscopie infrarouge à transformée de Fourier (FTIR). II en est de même 
pour les méthodes de spectrométrie de masse de type échanges de proton et de deutérium (HDX-MS) [18]. La structure quaternaire de l'anticorps (hétérodimère de 2 chaînes lourdes et légères) peut être confirmée par exemple par spectrométrie de masse.

\section{Mode d'action}

Le mode d'action des AcM est également spécifique de ces médicaments. Contrairement aux petites molécules dont l'activité repose sur un schéma de type clé-serrure, le mécanisme d'action des AcM est souvent complexe, reposant à la fois sur leurs domaines de liaison à l'antigène (présents dans le fragment Fab) et sur des fonctions effectrices attribuables à leur région Fc. Selon la cible, sa nature (extracellulaire, membranaire, etc.) et le format de l'AcM, les fonctions à explorer sont différentes. Même si la séquence primaire de l'AcM et du biosimilaire sont identiques, il n'est pas assuré que le biosimilaire induise la même réponse biologique que l'AcM original.

À l'inverse, il est aussi possible que le biosimilaire présente des caractéristiques physico-chimiques différentes mais une activité biologique similaire. Ainsi, Hassett et al. [15] ont montré que des biosimilaires de l'étanercept avec des attributs physico-chimiques différents présentaient la même affinité pour leur cible. Il est donc impératif d'associer une analyse physico-chimique à l'étude des fonctions biologiques des biosimilaires.

Comme indiqué dans le Tableau II, la caractérisation biologique d'un AcM met en jeu : 1) l'affinité de l'AcM pour sa cible et la modification de son activité biologique intrinsèque, et 2) les fonctions effectrices liées à la région Fc de l'AcM. L'analyse de ces dernières repose essentiellement sur des tests cellulaires faisant intervenir différents partenaires selon la fonction étudiée (cellules immunitaires pour caractériser l'ADCC (cytotoxicité cellulaire dépendante des anticorps) et I'ADCP (phagocytose dépendante des anticorps). Par ailleurs, la glycosylation des anticorps, qui peut être modifiée dans le cas d'un biosimilaire, joue un rôle important dans la CDC (cytotoxicité dépendante du complément) et l'ADCC. À titre d'exemple, l'affinité du CT-P10 (biosimilaire du rituximab) pour CD20 a été comparée à celle du rituximab par la méthode ELISA (enzyme-linked immunosorbent assay) [12]. Les résultats n'ont pas montré de différences notables, avec des concentrations efficaces médianes $\left(\varepsilon C_{50}\right)$ similaires. Le CT-P10 présentait des différences mineures de composition en glycanes (proportion de formes afucosylées et riches en mannose), qui n'avaient pas d'impact sur les activités CDC et ADCC. La situation est différente pour deux biosimilaires de l'infliximab (Flixabi ${ }^{\circledR}$ et Remsima ${ }^{\circledR}$ ). En dépit d'une affinité identique pour le TNF- $\alpha$ (tumor necrosis factor alpha), l'activité ADCC de ces trois molécules diffère : la forte proportion de formes afucosylées et riches en mannose du Flixabi ${ }^{\circledR}$ est associée à une plus forte activité d'ADCC in vitro en comparaison avec le Remsima ${ }^{\circledR}$ et le Remicade ${ }^{\circledR}$ [19].

Les fonctions effectrices des anticorps peuvent également être caractérisées indirectement en étudiant l'affinité de la région Fc pour ses partenaires moléculaires - les récepteurs de la région Fc (RFc $\gamma$ ) pour les fonctions d'ADCC/ADCP et le Clq pour l'activité $C D C$ - par résonance plasmonique de surface [20]. En accord avec les résultats des tests cellulaires des fonctions effectrices, l'analyse comparative de CT-P10 et du rituximab n'a pas révélé de différences d'affinité des ligands de la région $\mathrm{Fc}$ (Clq, RFcyllla-V/F, RFcylllb, RFcylla, RFc $\gamma l l b$ et FcRn) [12]. A contrario, les biosimilaires de l'infliximab ont montré des différences de comportement in vitro, notamment en ce qui concerne leur fixation au RFcyllla [19].

\section{Conclusion}

En conclusion, malgré la complexité structurale, physico-chimique et fonctionnelle des AcM, la réalisation de copies similaires est possible, mais des contrôles multiples et minutieux sont nécessaires pour établir cette similarité. L'analyse physico-chimique et fonctionnelle est la première étape de cette démonstration en comparaison au biomédicament princeps (le produit de référence) par des études in vitro qui nécessitent des investissements analytiques importants pour garantir cette biosimilarité. Une fois ces études préliminaires réalisées et validées, il faut envisager des études précliniques pour évaluer la pharmacodynamie et la toxicologie, puis des études cliniques de biosimilarité chez l'homme afin d'évaluer la pharmacocinétique (PK)/pharmacodynamie (PD), l'efficacité, la sécurité d'utilisation et l'immunogénicité au cours d'essais cliniques de phase III. La réalisation de ces études, coûteuses mais indispensables, a permis depuis 2013 I'autorisation en Europe de près de 30 biosimilaires des 6 anticorps monoclonaux ou protéine de fusion ayant les plus importants chiffres d'affaires, mais à un prix de vente inférieur à celui du biomédicament référent, offrant ainsi un accès à ces thérapies innovantes pour un plus grand nombre de patients. $\diamond$

\section{SUMMARY}

Biosimilar monoclonal antibodies: comparative study of analytical and functional quality

Biosimilars marketing authorization requires a strict demonstration of similarity with the reference antibody, through preclinical and clinical studies. This article reviews the panel of in vitro physicochemical and functional analyses, which are performed prior to clinical studies. For each critical attribute of the antibody, we detail the commonly used analytical techniques, their working principle and the type of information they may give. $\diamond$

\section{LIENS D’INTÉRÊT}

Les auteurs déclarent n'avoir aucun lien d'intérêt concernant les données publiées dans cet article. 


\section{RÉFÉRENCES}

1. Reichert JM, Beck A. European medicines agency workshop on biosimilar monoclonal antibodies. MAbs $2009 ; 1: 394-416$.

2. Beck A, Reichert JM. Approval of the first biosimilar antibodies in Europe: a major landmark for the biopharmaceutical industry. MAbs $2013 ; 5: 621-3$.

3. Ayoub D, Jabs W, Resemann A, et al. Correct primary structure assessment and extensive glycoprofiling of cetuximab by a combination of intact, middle-up, middle-down and bottom-up ESI and MALDI mass spectrometry techniques. MAbs $2013 ; 699-710$.

4. Gahoual R, Biacchi M, Chicher J, et al. Monoclonal antibodies biosimilarity assessment using transient isotachophoresis capillary zone electrophoresis-tandem mass spectrometry. MAbs $2014 ; 6$ : 1464-73.

5. Beck $A$, Wagner-Rousset $\varepsilon$, Wurch $T$, et al. Anticorps thérapeutiques et dérivés : une palette de structures pour une pléthore d'indications. Quel format et quelle glycosylation choisir? Pour quelles applications? Med Sci (Paris) $2009 ; 25: 1024-32$.

6. Beck A. Biosimilar, biobetter and next generation therapeutic antibodies. MAbs $2011 ; 3: 107-10$.

7. Beck A, Sanglier-Cianférani S, Dorsselaer A Van. Biosimilar, biobetter, and next generation antibody characterization by mass spectrometry. Anal Chem 2012 ; $84: 4637-46$.

8. Beck A, Debaene F, Diemer H, et al. Cutting-edge mass spectrometry characterization of originator, biosimilar and biobetter antibodies. J Mass Spectrom $2015 ; 50: 285-97$

9. Ambrogelly A, Gozo S, Katiyar A, et al. Analytical comparability study of recombinant monoclonal antibody therapeutics. MAbs $2018 ; 10: 513-38$.

10. Aerts L van, Smet K De, Reichmann G, et al. Biosimilars entering the clinic without animal studies A paradigm shift in the European Union. MAbs 2014; $6: 1155-62$.

11. Rahalkar H, Cetintas HC, Salek S. Quality, non-clinical and clinical considerations for biosimilar monoclonal antibody development: $\varepsilon U, W H O$, USA, Canada, and BRICS-TM regulatory guidelines. Front Pharmacol 2018 ; 9

12. Lee KH, Lee J, Bae JS, et al. Analytical similarity assessment of rituximab biosimilar CT-P10 to reference medicinal product. MAbs 2018; $10: 380-96$

13. Nupur N, Chhabra N, Dash R, et al. Assessment of structural and functional similarity of biosimilar products: Rituximab as a case study. MAbs $2018 ; 10: 143-58$
14. Lee $\mathrm{N}$, Lee JAJ, Yang $\mathrm{H}$, et al. Evaluation of similar quality attribute characteristics in SB5 and reference product of adalimumab. MAbs 2018 ; $11: 129-44$.

15. Hassett B, Scheinberg M, Castañeda-Hernández G, et al. Variability of intended copies for etanercept (Enbrel $\left.{ }^{\circledR}\right)$ : data on multiple batches of seven products. MAbs $2018 ; 10: 166-76$.

16. Beck A, Wagner-Rousset $\varepsilon$, Bussat MC, et al. Trends in glycosylation, glycoanalysis and glycoengineering of therapeutic antibodies and $\mathrm{Fc}$-fusion proteins. Curr Pharm Biotechnol $2008 ; 9: 482-501$.

17. Largy $\varepsilon$, Cantais $F$, Vyncht $G$ Van, et al. Orthogonal liquid chromatographymass spectrometry methods for the comprehensive characterization of therapeutic glycoproteins, from released glycans to intact protein level. J Chromatogr A $2017 ; 1498$ : 128-46.

18. Fang J, Doneanu C, Alley WRJ, et al. Advanced assessment of the physicochemical characteristics of Remicade ${ }^{\circledR}$ and Inflectra ${ }^{\circledR}$ by sensitive LC/MS techniques. MAbs 2016 ; 8 : 1021-34.

19. Lee C, Jeong M, Lee JAJ, et al. Glycosylation profile and biological activity of Remicade ${ }^{\circledR}$ compared with Flixabi® ${ }^{\circledR}$ and Remsima ${ }^{\circledR}$. MAbs 2017 ; 9 : 968-77.

20. Brachet $\mathrm{G}$, Respaud $\mathrm{R}$, Arnoult $\mathrm{C}$, et al. Increment in drug loading on an antibody-drug conjugate increases its binding to the human neonatal $\mathrm{Fc}$ receptor in vitro. Mol Pharm 2016; 13: 1405-12.

21. Seo N, Polozova A, Zhang M, et al. Analytical and functional similarity of Amgen biosimilar ABP 215 to bevacizumab. MAbs $2018 ; 10$ : 678-91

22. Liu J, Eris T, Li C, et al. Assessing analytical similarity of proposed amgen biosimilar ABP 501 to Adalimumab. BioDrugs 2016; 30 : 321-38.

23. Magnenata L, Palmeseb A, Fremauxc C ele, et al. Demonstration of physicochemical and functional similarity between the proposed. MAbs $2017 ; 9: 127-39$.

TIRÉS À PART

R. Respaud

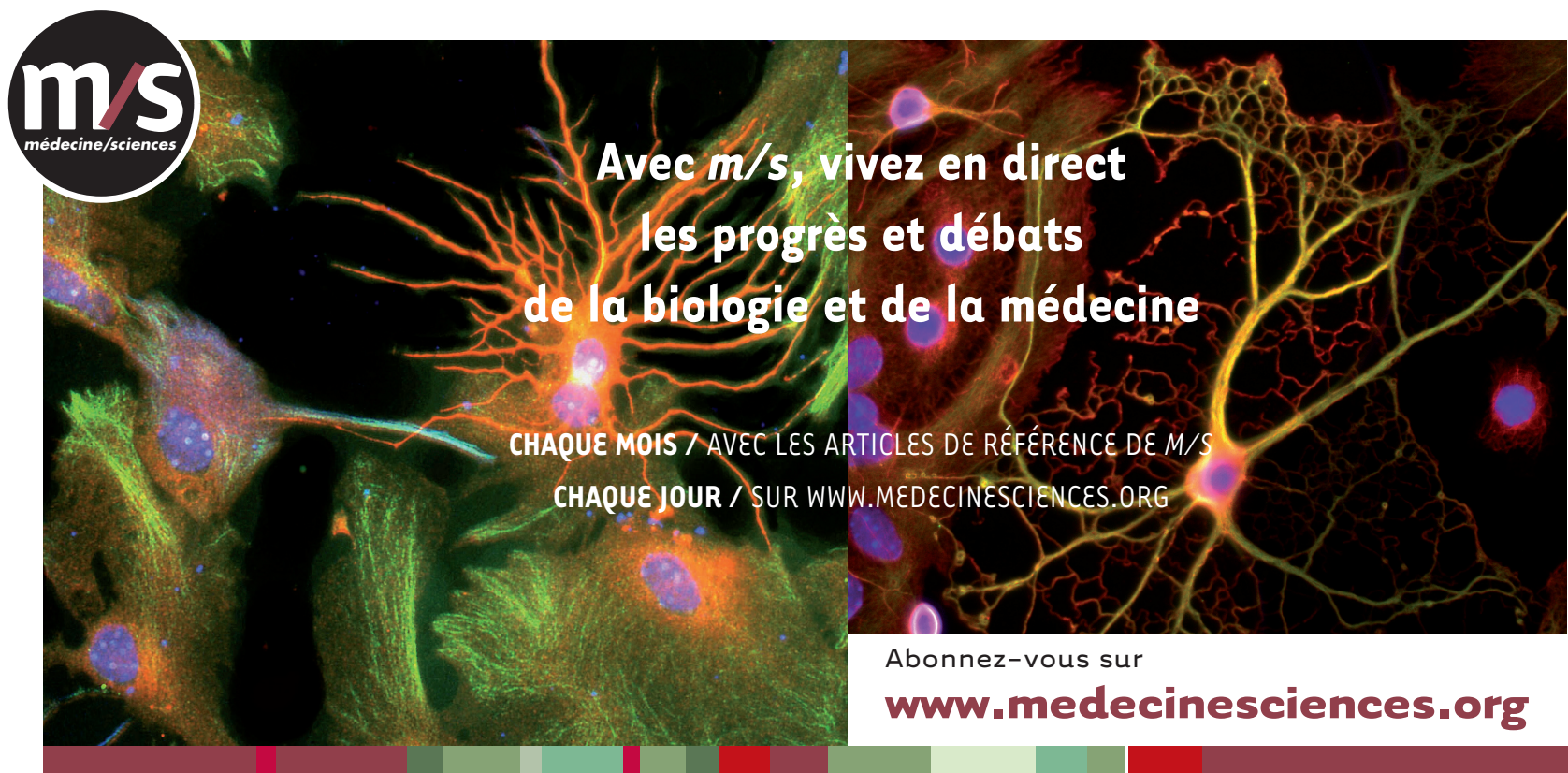

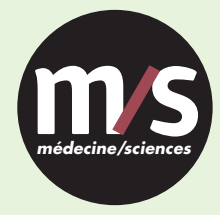

Tarifs d'abonnement $\mathrm{m} / \mathrm{s}-2019$

Abonnez-vous

à médecine/sciences
$>$ Grâce à $m / s$, vivez en direct les progrès des sciences biologiques et médicales

Bulletin d'abonnement page 1230 dans ce numéro de $\mathrm{m} / \mathrm{s}$

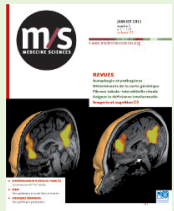

